\title{
High-throughput 3D Cellular Imaging
}

\author{
G. Knott, ${ }^{*}$ D. Wall ${ }^{* *}$ and B. Lich ${ }^{* *}$ \\ *Ecole Polytechnique Federale de Lausanne, 1015 Lausanne, Switzerland \\ ** FEI Company, Achtseweg Noord 5, 5651 GG Eindhoven, The Netherlands
}

Understanding complex biological systems requires knowing how cells are organized in a 3D network because their organization determines how cells can interact. Perhaps the most challenging example found in nature is the neuronal circuits within a volume of brain tissue. High resolution is required to see the smallest synaptic contacts, but at the same time a large volume of tissue must be imaged to understand the intercellular interactions.

Traditionally serial section transmission electron microscopy has been the only method available in which images are acquired from sections that are cut and mounted on grids. These images representing a 3D volume can be used to reconstruct and analyze the structure of the elements within. This method is very labor intensive, requiring a greater deal of manual dexterity, and when small mistakes occur, a continuous dataset is lost.

Recent studies have explored an alternative method for serial image acquisition in which the block face of resin-embedded neural tissue was imaged within a scanning electron microscope (SEM). Sections were removed from the imaged face in the microscope using an ultramicrotome and then immediately imaged by the SEM. This provides a series of aligned images of the tissue in the block face and has the clear advantage that image acquisition can be fully automated. However the quality of the sectioning is critically dependent on homogeneous resin hardness which is difficult to maintain when the electron beam is being used at higher resolution and part of the block suffers from the differential heating effects.

We have continued to explore an alternative approach that is seemingly unaffected by variations in resin quality that uses a focused ion beam (FIB) directed perpendicular to the block face. This is used to mill the surface of the resin from which serial images can be acquired. We have used automated repetitive cutting and imaging on the DualBeam with consistent sectioning intervals to as low as $10 \mathrm{~nm}$.

Results as shown in fig demonstrate that this FIB technique is capable of sectioning neural tissue and using the back scattered electrons we were able to visualize the detailed ultrastructure. Synaptic contacts were clearly visible, including the presynaptic and postsynaptic densities and the synaptic vesicles. In a fully automated mode we were able to collect serial images that allowed us to make detailed morphological analyses of the neuronal elements in 3D. Moreover, using Slice and View G2, slice thickness can be determined using the FIB image stack. This yields a thickness measurement for each individual slice after completing the automated run. We demonstrate that these slice thickness measurements can be used to determine important statistics of the stack such as average slice thickness, standard deviation and maximum and minimum slice thickness. A high degree of automation in the acquisition and reconstruction steps allows this technique to be routinely applicable to the study of even the most complex tissue organizations. 

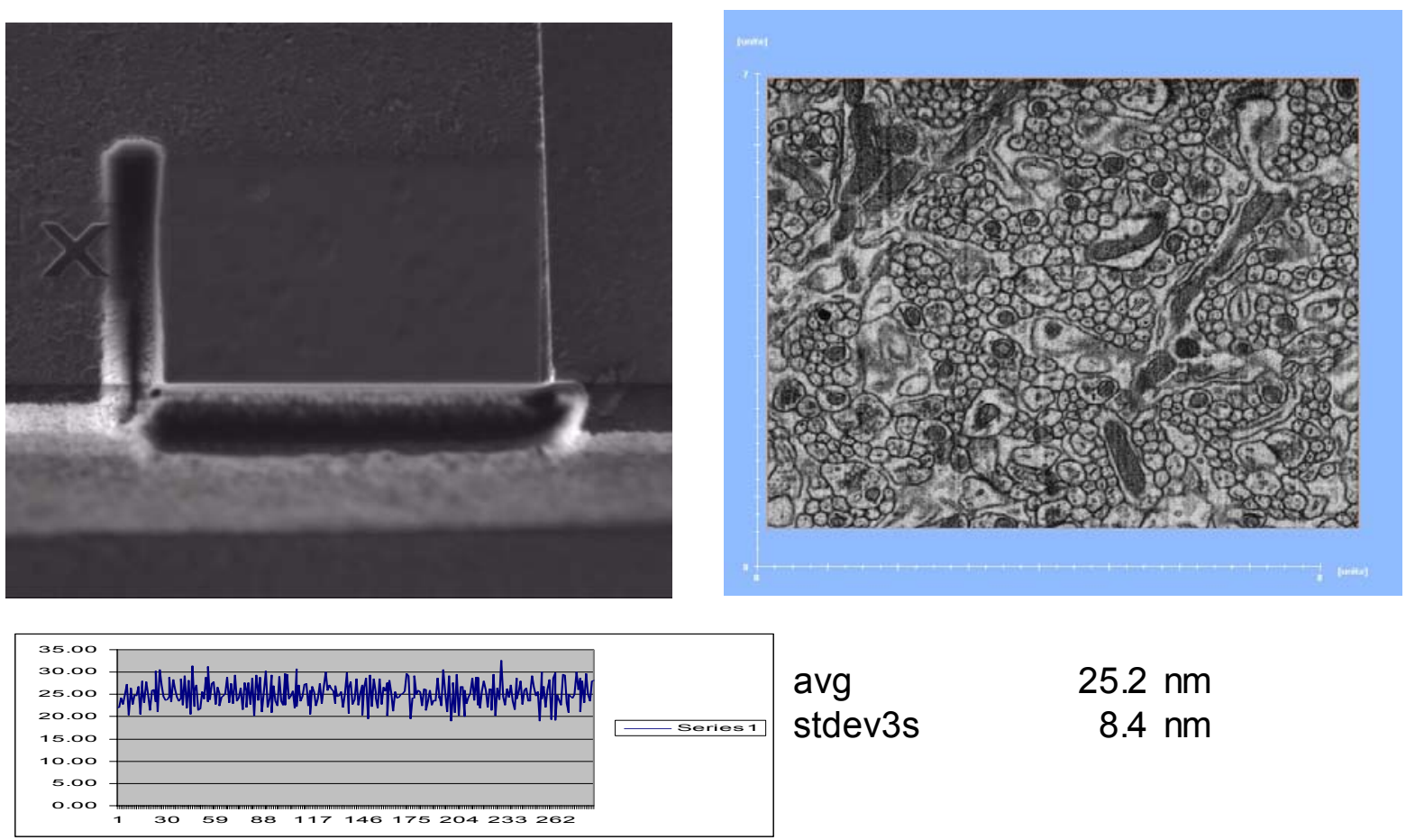

avg

stdev3s
$25.2 \mathrm{~nm}$

$8.4 \mathrm{~nm}$

Fig 1 - left top : Top view FIB image; right top : SEM image of the tissue, Bottom : slice thickness per slice 